Revista do SELL

v. 5 , no. 1

ISSN: 1983-3873

\title{
TROUBLE E PROBLEM: VERIFICAÇÃO DA CORRESPONDÊNCIA DOS SINÔNIMOS EM CORPORA
}

TROUBLE AND PROBLEM: CHECKING THE CORRESPONDENCE OF

SYNONYMS IN CORPORA

\author{
Eliene de Souza Paulino \\ Universidade Federal de Minas Gerais
}

\begin{abstract}
RESUMO: O presente artigo discute o conceito de "sinônimos" na língua por meio da comparação de trouble e problem em suas ocorrências no Corpus of Contemporary American English. Para tratar do assunto recorremos aos colocados e à prosódia semântica das duas palavras em estudo, pois entendemos que, gramaticalmente, pode não haver impedimento no intercâmbio das mesmas, mas para efeito de sentido é necessário que se verifique em quais contextos cada uma delas seria mais utilizada e, portanto, mais adequada. A Linguística de Corpus apresenta ferramentas que nos auxiliam nessas e em outras questões, desenvolvidas nessa pesquisa que tem a intenção de responder: "Quais são as semelhanças e diferenças no uso dos vocábulos "trouble e problem"?" "Essas palavras atuam como sinônimas?". Para chegarmos às conclusões, foram conferidos o sentido das palavras em diferentes contextos por meio das linhas de concordância, a frequência na língua, as palavras a elas associadas, os registros nos quais as palavras são mais comuns e a padronização no uso. Como resultado, a pesquisa corrobora o pressuposto o de Sinclair (1991), pois problem e trouble podem ocorrer com sentidos diferentes associadas a um conjunto distinto de padrões. Análises baseadas em corpus contribuem para revelar tais diferenças nas palavras consideradas sinônimas.
\end{abstract}

PALAVRAS-CHAVE: Linguística de Corpus; sinônimos; trouble; problem

ABSTRACT: This study discusses the concept of synonyms by comparing "trouble" and "problem" in the Corpus of Contemporary American English. For the purpose of the study, the semantic prosody and collocations of the words were analyzed, under the consideration that even if there isn't any grammatical constraint in using those words in different sentences, singular contexts require "trouble" instead of "problem," and vice versa, to create a different effect or meaning, which suggests a difference in use. Corpus Linguistics methodology includes tools that can be used in these types of research and others. This study intends to answer: "What are the similarities and differences in the use of 'trouble' and 'problem' in the language?" and "Do the words work as synonyms?" In order to arrive at our conclusions, the meaning of the words in different contexts were studied in the corpus-generated concordance lines, as well as the frequency of the words, the words associated with them, their use in different registers and patterns of use. This study confirms that each of the words studied occurs with singular meanings associated with a different set of patterns (SINCLAIR, 1991). Corpus-based analysis contributes to the detection of those differences in words considered as synonyms.

KEYWORDS: Corpus Linguistics; synonyms; trouble; problem

\section{Considerações Iniciais}

$\mathrm{Na}$ escola os sinônimos são ensinados sob a justificativa de aprimorar o vocabulário do estudante, mostrá-los outras possibilidades de uso de palavras para originar significado semelhante ou parecido e, entre outras coisas, como estratégia para 


\section{Revista do SELL \\ v. 5 , no. 1 \\ ISSN: $1983-3873$}

evitar a repetição de termos em um texto. A Linguística de Corpus, segundo Römer (2009), possui um grande potencial para auxiliar e melhorar a prática pedagógica. Uma das formas seria apontar caminhos alternativos aos professores para que sejam auxiliados nas tarefas do dia-a-dia através de corpora no ensino.

Do mesmo modo, enquanto pesquisadores, recorremos aos sinônimos nos nossos discursos a fim de tornar a comunicação mais fluida e sofisticada. Em muitas referências os sinônimos são descritos como palavras que se substituem e possuem o mesmo sentido. Mas será que é assim mesmo? Quando os estudantes nos questionam qual é a diferença entre duas palavras ditas "sinônimas", sabemos o que responder ou onde buscar respostas? Diante de tal indagação torna-se oportuno o estudo do uso autêntico de palavras e expressões com base em corpora, e, para tratar do assunto, recorremos aos colocados das palavras, pois entendemos que gramaticalmente pode não haver impedimento no intercâmbio das palavras, mas para efeito de sentido, o uso equivocado pode causar estranheza, como em *We're in problem”, para a qual não há ocorrência no corpus, ao passo que "We're in trouble” é uma expressão habitual na língua.

Todos estamos familiarizados com a situação em sala de aula na qual recorremos a afirmação 'é porque é assim que se diz', quando confrontados com uma pergunta embaraçosa de um estudante sobre porque algo é dito de tal forma, e, muitas vezes, o que estamos de fato explicando é uma forte preferência estatística que pode ser poderosamente demonstrada pela utilização de dados de corpus. A resposta, em última análise, é ainda que a colocação nos mostra 'a forma como dizer', mas ganharemos uma confiança considerável como professores se pudermos apresentar algo como uma colocação difundida e frequente ao invés de uma única ocorrência em um texto particular no qual estamos trabalhando. ${ }^{1}$ (O'KEEFFE, MCCARTHY \& CARTER, 2007, p. 59)

A Linguística de Corpus apresenta ferramentas que nos auxiliam nessas e em outras questões, as quais pretendemos desenvolver no presente estudo que tem a intenção de responder: "Quais são as semelhanças e diferenças no uso dos vocábulos "trouble e problem"?" e com isso, verificar se essas palavras atuam como sinônimas. Para

1

We are all familiar with the situation in class where we fall back on the statement 'that's just the way we say it', when faced with an awkward question from a student about why something is expressed the way it is, and often, what we are really explaining is a strong statistical preference which can be powerfully demonstrated by the use of corpus data. The answer, in the final analysis, is still that collocation shows us 'the way we say it', but we can gain considerable confidence as teachers if we can present something as a widespread and frequent collocation rather than a one-off occurrence in the particular text we are working with. (O'KEEFFE, MCCARTHY \& $\quad$ CARTER, 


\section{Revista do SELL \\ v. 5 , no. 1 \\ ISSN: $1983-3873$}

chegarmos às conclusões, foram verificados a frequência das palavras na língua, o sentido das palavras em diferentes contextos por meio das linhas de concordância, palavras associadas a cada uma delas, em quais registros as palavras são mais comuns e se há padronização no uso, ou seja, palavras que tendem a ocorrer com uma ou outra. A seguir discutirei brevemente os pressupostos teóricos relacionados à Linguística de Corpus como colocados e prosódia semântica, os passos metodológicos para a realização da pesquisa, a análise dos dados, resultados e conclusões.

\section{Pressupostos Teóricos}

Nesta seção apresentamos a fundamentação teórica da pesquisa, a começar com uma breve definição da Linguística de Corpus (LC) e, em seguida, trataremos de colocações e língua como fraseologia e prosódia semântica, tendo como embasamento Sinclair (1991); Biber, Conrad \& Reppen (1998); Hunston (2002); Sardinha (2004) e Bennett (2010), como norteadores da discussão.

\subsection{A Linguística de Corpus (LC)}

A LC tem como pressuposto o estudo da língua em uso por meio do uso de dados autênticos compilados em corpus. (BENNETT, 2010). Segundo Sardinha (2004), a metodologia ocupa-se da coleta, armazenamento e estudo dos dados obtidos. O vasto material linguístico que compõe os corpora são coletados com o propósito específico da pesquisa de uma língua ou variedade linguística, que se destina a evidências empíricas extraídas do computador. "A Linguística de Corpus é a é a área que trata dos estudos que utilizam corpora eletrônicos em suas análises" (DUTRA, 2009). Por corpus, entende-se a coleção de dados linguísticos autênticos, obtidos no seu contexto natural de produção, passíveis de leitura pelo computador e selecionados por meio de critérios externos, com objetivos particulares e pré-definidos. A criação de um corpus está voltada para a finalidade a que ele se propõe e, por meio dele, a língua pode ser vista em contextos particulares, inacessíveis somente à atitude intuitiva. Um corpus traz evidência linguística para o que antes era atribuído à intuição (O'KEEFFE, MCCARTHY e CARTER 2007). 


\section{Revista do SELL}

v. 5 , no. 1

ISSN: $1983-3873$

A abordagem da LC é empírica, uma vez que trata dos padrões da língua observados em contextos naturais (BIBER et al. 1998). Nesses contextos, uma série de investigações pode ser realizada, entretanto, nos detemos aqui em algumas delas, como o significado e uso das palavras, definido como lexicografia. Por esse prisma são estudados os sinônimos, o que há de comum entre palavras diferentes, quão comuns são os diferentes sentidos de uma mesma palavra e as associações entre elas. O estudo lexicográfico baseado em corpus estabelece "(...) como palavras relacionadas são usadas de formas diferentes e apropriadas em diferentes contextos."2 (BIBER et al. 1998,p. 21).

Os autores supracitados apontam que a frequência da ocorrência das palavras nos corpora permite o entendimento dos padrões de uso associados a cada uma delas. Para Sinclair (1991), se uma palavra tem mais de um significado, cada um deles tende a ser associado a um conjunto diferente de padrões.

\subsection{Colocações e Prosódia Semântica}

Algumas palavras tendem a ocorrer juntas na língua, não havendo, para tanto, regras linguísticas que expliquem a escolha da companhia de certas palavras. $\mathrm{O}$ fato é que o uso recorrente de algumas expressões fez com que as mesmas passassem a ser consideradas uma padronização da língua. Para Sinclair (1999) os usuários da língua contam com um grande número de frases semi-construídas as quais estão à disposição do falante. Sendo assim, as escolhas passam a ser feitas pela unidade de sentido que as frases carregam. A tendência de algumas palavras simplesmente ocorrerem juntas na língua é chamada de colocação.

Com o intuito de elucidar como o sentido é originado do texto, Sinclair (1991) sinaliza dois tipos de interpretação, um deles, no qual nos deteremos no presente trabalho, é o princípio idiomático, que parte da hipótese de que a organização da língua contribui para construções menos imprevisíveis, ou seja, algumas palavras comumente aparecem em combinações pré-estabelecidas pelos usuários, as quais apresentam uma frequência relevante no uso, são as expressões fixas, pares ou grupos de palavras que

2

"(...) how related words are used in different ways and are appropriate in different contexts." BIBER; CONRAD \& REPPEN 1998: 21) 


\section{Revista do SELL \\ v. 5 , no. 1 \\ ISSN: 1983-3873}

sempre aparecem juntos. Para exemplificar, tomemos o par: "merry birthday", o qual não configura uso real da língua, pois a tendência é o uso de "happy birthday". Observando os dois pares nota-se que, gramaticalmente, não há problemas em ambos, contudo, o uso do primeiro par causa estranheza, pois não representa o que um falante nativo diria. Utilizando os mesmos adjetivos podemos exemplificar o uso de Merry para Christmas, o qual é significativamente mais usado que happy, nesse contexto.

O estudo das frases (fraseologia) atua como elemento central na Linguística de Corpus. Nesse campo está incluído o estudo das colocações, pacotes lexicais e sequências preferenciais. A tendência de palavras coocorrerem é definida como collocation (BENNETT, 2010). Cunhado pelo linguista britânico Firth (1957, apud ALMEIDA, 2014) o termo collocation tornou-se conhecido e estudado por outros pesquisadores desde então, que, de modo geral, permanece relacionado à consideração de que o significado de uma palavra está atrelado às palavras à sua volta. É possível, por meio das análises em corpora, descobrir padrões de ocorrência das palavras que comumente surgem juntas no uso da língua. O'Keeffe, McCarthy e Carter (2007), retomam Firth (1935) para confirmar que o sentido de uma palavra está mais relacionado a como esta palavra é combinada com outras em seu uso do que ao significado que ela possui em si mesma, "Colocações não são absolutas ou determinantes, mas são eventos probabilísticos, os quais resultam de combinações repetidamente utilizadas e encontradas por falantes de qualquer língua" (O'KEEFFE ET AL. 2007, p. 59). ${ }^{3}$

Um corpus pode auxiliar no aperfeiçoamento do uso da língua através de sua grande amostra de colocações e da possibilidade de visualização dos padrões léxicogramaticais, os quais comprovam que a língua não é formada por palavras individuais, mas que seu vocabulário possui "unidades pré-fabricadas" (Sardinha, 2006), como em: make a mistake, have breakfast e take a shower. Barlow (2004), com base em (Barlow e Kemmer, 2000; Bybee e Hopper, 2001; Firth 1957 e Sinclair 1991) explica que o interesse dos linguistas nas colocações é ilustrado pelo entendimento de que as colocações funcionam como blocos de sentido, os quais refletem a língua em uso, e, além disso, o contexto em volta das palavras em estudo fornece informações sobre as particularidades

3

Collocations are not absolute or deterministic, but are probabilistic events, resulting from repeated combinations used and encountered by the speakers of any language. (O'KEEFFE ET AL. 2007, p. 59). 


\section{Revista do SELL}

v. 5 , no. 1

ISSN: 1983-3873

de uso da palavra e sobre o funcionamento da língua, o que contribui na organização da gramática.

Sardinha (2000) elenca as três principais definições de colocação na literatura, segundo Partington (1998), colocação textual, psicológica e estatística, nas quais são verificadas a ocorrência das palavras, o sentido colocacional e a probabilidade de itens lexicais ocorrerem no seu contexto, respectivamente. O estudo do contexto em uso dá origem ao significado conotacional do léxico, o termo conotação é usado para fenômenos distintos, uma vez que a conotação pode ser social ou situacional, sofrendo a influência de classe, origem e sexo dos falantes, por exemplo. Sendo assim, aspectos culturais interferem nos significados estabelecidos para as palavras dentro de seus contextos diversos (PARTINGTON, 1998).

O estudo dos colocados permite, após a análise das linhas de concordância, que conclusões acerca do ambiente semântico das palavras possam ser registradas. A associação entre itens lexicais e as conotações negativa, positiva e neutra são identificados como prosódia semântica. Hunston (2002) alega que a prosódia semântica é um importante aspecto da língua, contudo, nem sempre faz parte do conhecimento consciente do falante e tampouco é ensinada aos aprendizes. Para ela, a associação de padrões e significado é de tal maneira relevante que faz com que o sentido pertença à frase como um todo e não às palavras individuais que a compõem. O termo prosódia semântica é usado para descrever essa situação, "Refere-se, geralmente, a uma palavra que é tipicamente usada em um ambiente particular, de tal forma que a palavra passa a ter conotações daquele ambiente" (SINCLAIR 1991; LOUW 1993, 1997, STUBBS 1996; HUNSTON 1995ㅜ; CHANNELL 2000, APUD HUNSTON 2002). Todavia, antes que se faça alguma generalização no tocante à prosódia semântica de um item lexical, um grande número de ocorrências de uma palavra ou frase deve ser observado, para que assim seja possível fazer qualquer afirmação sobre o uso típico ou padronizado.

Partington (1998), lembra que a expressão "semantic prosody" foi evidenciada por Sinclair em 1987 para descrever o fenômeno da associação de palavras à eventos

4

"It usually refers to a word that is typically used in a particular environment, such that the word takes on connotations from that environment" (SINCLAIR 1991; LOUW 1993, 1997, STUBBS 1996; HUNSTON 1995"; CHANNELL 2000, APUD HUNSTON 2002) 


\section{Revista do SELL}

v. 5 , no. 1

ISSN: $1983-3873$

desagradáveis por causa da "má companhia" das palavras que a cercam, tendo, portanto, prosódia desfavorável ou, por outro lado, prosódia positiva e até mesmo neutra.

As colocações e a identificação da prosódia semântica das palavras nos auxiliam na discussão dos sinônimos. As línguas possuem inúmeras palavras consideradas sinônimas e idênticas em termos de sentido. Contudo, Biber, Conrad \& Reppen (1998, p.43) comprovam que "palavras "sinônimas" são geralmente usadas de maneiras muito diferentes"5 e que as análises baseadas em corpus contribuem para revelar as diferenças nos padrões de uso.

Para Tsui (2004) saber se há diferença entre palavras reconhecidas como sinônimas é uma indagação recorrente e, por vezes, a diferença é notável, todavia, não se sabe explicar como se dá essa diferença. Por exemplo, para explicar a diferença entre high e tall, Tsui (2004) cita as estratégias utilizadas por dois professores. Um deles, preferiu dar o exemplo de uma frase, já o outro professor, recorreu à definição em um dicionário para explicitar a diferença no uso. A solução mais viável para o questionamento, por fim, veio de um corpus, no qual foi possível observar os um grande número de substantivos modificados por high e tall e concluir, entre outras coisas, que high é mais utilizado no sentido metafórico (high industrial growth, high concentration, high priority, high price) e tall é utilizado para substantivos concretos (tall building, tall man, tall tree) (TSUI, 2004). Tudo isso serve para ilustrar a proposição de que uma palavra não pode ser entendida isoladamente, mas a partir da observação de suas preferências semânticas (SINCLAIR, 1991).

Outra discussão a respeito dos sinônimos das palavras é dirigida por Biber, Conrad \& Reppen (1998), os quais exemplificaram como big, large e great, embora sejam comumente caracterizadas como palavras sinônimas de size, após as investigações dos vários usos de great relativos a big e large demonstram que cada adjetivo tem seus colocados, sentidos e distribuições preferenciais nos registros. Já os adjetivos horrible e terrible são citados por O'Keeffe et al. (2007) pois, embora tenham significados próximos, horrible caracteriza pessoas, coisas e situações de forma mais subjetiva, como horrible

5 1998, p.43)

"synonymous" words are typically used in very different ways."(BIBER, CONRAD \& REPPEN, 


\section{Revista do SELL \\ v. 5 , no. 1 \\ ISSN: $1983-3873$}

smell, ao passo que terrible é utilizado para situações mais objetivas, como terrible earthquake.

Em suma, a observação dos colocados e da prosódia semântica e as conclusões a respeito da diferença no comportamento das palavras na língua, bem como os efeitos de sentido gerados por elas, auxiliam-nos a revelar aspectos relacionados às palavras ditas sinônimas.

\section{Procedimentos Metodológicos}

Para o presente estudo será considerado o par de palavras trouble e problem. A pesquisa terá como base análises feitas a partir dos colocados e das linhas de concordância geradas pelo Corpus of Contemporary American English (COCA ${ }^{6}$. A seguir será apresentado, de forma breve, o corpus utilizado e os procedimentos de coleta e análise dos dados.

\subsection{Corpus COCA}

Com 450 milhões de palavras em 189. 431 textos, incluindo 20 milhões de palavras a cada ano, de 1990 a 2012, compreendendo os registros acadêmico, revista, jornal, conversação e ficção, Corpus of Contemporary American English (COCA, (DAVIES, 2008)), disponibilizado para consultas gratuitas após cadastro, foi escolhido para essa pesquisa.

\subsection{Descrição da Coleta e Análise}

Os dados apresentados nesse artigo foram coletados em diferentes etapas. Primeiro, foi feita a pesquisa de cada palavra a fim de averiguar a sua frequência no corpus e nos registros. Depois, foram selecionados os 20 primeiros colocados do primeiro nódulo à esquerda e à direita, nas posições 1, 2, 3 e 4 e a busca pelos sinônimos. 0 mesmo trabalho foi feito para a segunda palavra.

Depois de compilar e analisar os primeiros dados, obtivemos as 50 primeiras linhas de concordância das palavras trouble e problem e verificamos as suas ocorrências nos diferentes contextos. Para maior aprofundamento da análise, foi feita a escolha de 


\section{Revista do SELL \\ v. 5 , no. 1 \\ ISSN: 1983-3873}

verificar o contexto expandido de algumas linhas de concordância com a intenção de verificar os parâmetros construídos no uso real da língua.

Em termos estruturais e sintáticos, observamos quais palavras seguem ou antecedem trouble e problem. No que diz respeito à pragmática e prosódia semântica, foi observado como o contexto linguístico contribui para o sentido das palavras. Tendo isso em mãos, a sequência do estudo se deu com a investigação das linhas de concordância de cada palavra. Foram examinadas, em média, de 50 linhas para cada uma delas. Verifica-se que um estudo mais aprofundado poderia ser feito com todas as linhas de concordância de cada uma das palavras, entretanto, para a presente investigação, o número apresentado se fez suficiente.

Depois de feitas as análises citadas, foram explorados, brevemente, outros recursos oferecidos pela plataforma do corpus, como a lista de sinônimos gerada por ele. Uma análise apurada de alguns termos foi feita para chegar à conclusão, com base na pesquisa do uso, de como certas palavras são usadas e em quais contextos elas mais aparecem.

\section{Análise das palavras nos Corpora}

\subsection{Colocados de trouble}

A palavra trouble aparece com 41.182 ocorrências no corpus COCA. Destas ocorrências, o maior número é proveniente da ficção, com 11.969 ocorrências e o menor número procede da escrita acadêmica, com 2.605 ocorrências.

A palavra trouble é imediatamente seguida da palavra with 3.132 vezes, com o sentido de "ter problemas com algo ou alguém", trouble with my parents, trouble with drugs, etc. Após a preposição with, portanto, há a tendência de se usar um substantivo. Seguida imediatamente da palavra getting (764 vezes), com o sentido de "problemas para se obter algo", trouble getting financing, trouble getting a job, trouble getting pregnant, etc. Neste caso, logo em seguida, observa-se o uso de substantivos, substantivos formados por verbo seguido de -ing (financing) e adjetivo. 


\section{Revista do SELL}

v. 5 , no. 1

ISSN: $1983-3873$

Em relação às palavras que antecedem trouble, obtemos a preposição in, com 6.768 ocorrências. In trouble evidencia a ideia de estar em apuros, estar com problemas, sejam emergenciais ou não (got in trouble for it, she was in trouble, definitely in trouble).

Com base na lista de colocados verificados nas posições 1, 2, 3 e 4 apresentada abaixo, conclui-se que à esquerda há alta ocorrência dos verbos: have, got, get, stay, cause; advérbios: more, much, no, into; pronome: any; adjetivos: lot, big, serious, little, deep, foul, financial, entre outros. Observa-se o uso de adjetivos e intensificadores. À direita, nas posições 1, 2, 3 e 4 obtemos verbos como: getting, finding, keeping, sleeping, making, breathing, remembering, etc.; o advérbio ahead; a preposição: with e o substantivo spots. É perceptível que à direita de trouble há ocorrência significativa de verbos seguidos da partícula -ing (trouble getting a job, trouble understanding him, etc.) indicando propósito ou finalidade de algo, caso que, na língua portuguesa, recebe a preposição para antes dos verbos: problema para encontrar trabalho, problema para dormir. Para trouble to, houve 693 ocorrências. Em muitas delas, sequenciadas por verbos: trouble to engrave, trouble to be, trouble to send, trouble to answer, trouble to run.

Quadro 1: Colocados à esquerda e à direita de trouble

\begin{tabular}{|c|c|c|c|c|c|c|c|c|}
\hline & \multicolumn{4}{|c|}{ Colocados à esquerda de trouble } & \multicolumn{4}{|c|}{ Colocados à direita de trouble } \\
\hline & 1 & 2 & 3 & 4 & 1 & 2 & 3 & 4 \\
\hline 1 & $\ln$ & Have & have & into & with & getting & getting & getting \\
\hline 2 & Have & $\mathrm{Had}$ & into & having & getting & finding & finding & finding \\
\hline 3 & $\mathrm{Had}$ & Into & having & get & finding & keeping & keeping & keeping \\
\hline 4 & $\begin{array}{l}\text { Havin } \\
\mathrm{g}\end{array}$ & Having & get & got & spots & spots & spots & spots \\
\hline 5 & Into & No & much & lot & keeping & sleeping & sleeping & sleeping \\
\hline 6 & No & Get & any & big & sleeping & making & making & breathing \\
\hline 7 & Much & Much & got & serious & making & breathing & breathing & trouble \\
\hline 8 & Any & Any & lot & cause & breathing & $\begin{array}{l}\text { understandin } \\
\mathrm{g}\end{array}$ & $\begin{array}{l}\text { understandin } \\
\mathrm{g}\end{array}$ & $\begin{array}{l}\text { understandin } \\
\mathrm{g}\end{array}$ \\
\hline 9 & More & Lot & big & getting & understanding & seeing & seeing & seeing \\
\hline 10 & Big & Big & serious & deep & seeing & believing & believing & believing \\
\hline 11 & $\begin{array}{l}\text { Seriou } \\
\mathrm{s}\end{array}$ & Got & cause & real & began & ahead & ahead & remembering \\
\hline 12 & Little & Serious & getting & gets & believing, & $\begin{array}{l}\text { rememberin } \\
\mathrm{g}\end{array}$ & $\begin{array}{l}\text { rememberin } \\
\mathrm{g}\end{array}$ & spot \\
\hline 13 & Deep & Little & deep & gotten & started & spot & spot & brewing \\
\hline 14 & Real & Deep & real & stay & remembering & brewing & brewing & paying \\
\hline 15 & Cause & Real & gets & worth & ahead & paying & paying & staying \\
\hline 16 & $\begin{array}{l}\text { Finan } \\
\text { cial }\end{array}$ & Cause & $\begin{array}{l}\text { financi } \\
\text { al }\end{array}$ & $\begin{array}{l}\text { financi } \\
\text { al }\end{array}$ & spot & hearing & hearing & accepting \\
\hline 17 & $\begin{array}{l}\text { Enoug } \\
\mathrm{h}\end{array}$ & Getting & worth & $\begin{array}{l}\text { causin } \\
\mathrm{g}\end{array}$ & paying & staying & staying & paradise \\
\hline 18 & Heart & Kind & stay & sign & reading & walking & walking & $\begin{array}{l}\text { concentratin } \\
\mathrm{g}\end{array}$ \\
\hline 19 & Foul & Financi & gotten & signs & hearing & accepting & accepting & controlling \\
\hline
\end{tabular}




\section{Revista do SELL}

v. 5 , no. 1

ISSN: 1983-3873

\begin{tabular}{|c|c|c|c|c|c|c|c|c|}
\hline & & al & & & & & & \\
\hline 20 & $\begin{array}{l}\text { Causi } \\
\text { ng }\end{array}$ & Worth & $\begin{array}{l}\text { causin } \\
\mathrm{g}\end{array}$ & caused & staying & $\begin{array}{l}\text { concentratin } \\
\mathrm{g}\end{array}$ & $\begin{array}{l}\text { concentratin } \\
\text { g }\end{array}$ & adjusting \\
\hline
\end{tabular}

Fonte: Dados do corpus Corpus of Contemporary American English - elaborado pela autora (2015).

O nódulo aparece como substantivo na maioria dos casos do corpus, contudo, é possível que a palavra seja usada como verbo (335 exemplos), precedido da preposição to, como em:

\footnotetext{
clicks through a folder of photos his son emailed from Iraq, shots of him and his buddies. JOAN (O.S.) But , \&; JOAN'S BEDROOM -- NIGHT Joan sits on the edge of the bed; speaks into the phone. JOAN Hank? 20. CC hangs up. Hank stops on a photo that seems to trouble him, we don't see it. EXT. COPY PLACE -- NIGHT C Mike's photos spit out. Most of them are just snaps of him and his buddies. One of them is different -- Hank distance. The image is chilling. FADE
}

Figura 4: Contexto expandido de to trouble. Fonte: Corpus of Contemporary American English

\subsection{Colocados de problem}

A palavra problem possui 137. 526 ocorrências no COCA. O nódulo foi utilizado mais vezes na língua falada (43.434) e menos vezes na ficção. $\mathrm{Na}$ análise dos 20 primeiros colocados à esquerda e à direita, obtemos:

Quadro 2: Colocados à esquerda e à direita de problem

\begin{tabular}{|c|c|c|c|c|c|c|c|c|}
\hline & \multicolumn{4}{|c|}{ Colocados à esquerda de problem } & \multicolumn{4}{|c|}{ Colocados à direita de problem } \\
\hline & 1 & 2 & 3 & 4 & 1 & 2 & 3 & 4 \\
\hline 1 & This & No & solve & solve & is & solving & solving & solving \\
\hline 2 & No & Solve & real & part & with & behavior & behavior & solved \\
\hline 3 & Big & Real & part & real & solving & behaviors & solved & behavior \\
\hline 4 & Serious & Big & big & big & here & facing & behaviors & behaviors \\
\hline 5 & Biggest & serious & serious & serious & behavior & solved & facing & facing \\
\hline 6 & Another & another & major & solution & behaviors & lies & lies & solution \\
\hline 7 & Major & Major & biggest & major & areas & finding & worse & lies \\
\hline 8 & Same & biggest & solution & biggest & facing & arises & arises & solve \\
\hline 9 & Same & Drug & address & address & lies & exists & exists & arises \\
\hline 10 & Drug & address & drug & drug & solvers & solvers & solution & exists \\
\hline 11 & health & Main & solving & solving & arises & solver & solve & resolved \\
\hline 12 & Main & Fix & fix & solved & solver & solve & solvers & solvers \\
\hline 13 & Bigger & common & solved & fix & exist & faced & solver & solver \\
\hline 14 & Common & Solved & main & main & solved & $\begin{array}{l}\text { identificatio } \\
n\end{array}$ & faced & addressed \\
\hline 15 & $\begin{array}{l}\text { fundame } \\
\text { ntal }\end{array}$ & Solving & huge & huge & become & $\begin{array}{l}\text { compounde } \\
\text { d }\end{array}$ & resolved & acute \\
\hline 16 & Huge & Huge & bigger & bigger & worse & occurs & $\begin{array}{l}\text { compounde } \\
\text { d }\end{array}$ & $\begin{array}{l}\text { compounde } \\
\text { d }\end{array}$ \\
\hline 17 & Potential & Bigger & $\begin{array}{l}\text { fundament } \\
\text { al }\end{array}$ & $\begin{array}{l}\text { fundament } \\
\text { al }\end{array}$ & drinkers & drinkers & acute & stems \\
\hline 18 & $\begin{array}{l}\text { Particula } \\
r\end{array}$ & $\begin{array}{l}\text { fundament } \\
\text { al }\end{array}$ & basic & root & identification & stems & occurs & arose \\
\hline 19 & Drinking & potential & resolve & resolve & stems & arose & drinkers & drinkers \\
\hline 20 & Difficult & particular & drinking & drinking & faced & posed & arose & posed \\
\hline
\end{tabular}

Fonte: Dados do corpus Corpus of Contemporary American English - elaborado pela autora (2015). 


\section{Revista do SELL \\ v. 5 , no. 1 \\ ISSN: 1983-3873}

Nos colocados à esquerda, observamos que alguns verbos se configuram no passado e presente e alguns adjetivos se repetem nas colunas (tanto da direita quanto da esquerda). Com base na lista de colocados verificados nas posições, observa-se à esquerda a ocorrência dos verbos: solve, address, fix, resolve; pronome: this; adjetivos: real, big, serious, major, main, common, huge, fundamental, potencial, particular, drinking, basic, difficult e substantivos como: part, solution, drug, root.

À direita obtemos os verbos solve, is, face, lie, exist, arise, compound, posed, occurs e stem; o advérbio here; a preposição with; os substantivos behavior, area, solution, drinkers e identification e o adjetivo: worse.

O substantivo problem é seguido pelo verbo is 14.024 vezes no corpus COCA, em frases que tem por objetivo especificar algum impedimento ou dificuldade e tecer comentários sobre situações incômodas que precisam ser resolvidas, "the underlying problem is simply that the otters are running out of food".

Uma das observações relevantes sobre os verbos que o antecedem, é a conotação de resolução concatenada a eles (fix, solve, resolve). Além disso, à esquerda de problem há uma tendência considerável do uso de adjetivos. Verbos, substantivos e adjetivos tanto precedem quanto sobrevêm problem. Nos exemplos em análise, à esquerda ocorrem pronomes e à direita preposição e advérbio.

\subsection{Comparação dos colocados de trouble e problem}

O estudo da lista de colocados oferece elementos para a configuração da padronização de palavras. De acordo com Sardinha (2011), "Os colocados mostram a padronização do nódulo, que, por sua vez, revela como essa palavra é usada, em termos de suas colocações, coligações e prosódia semântica."

Como é possível atestar por meio dos quadros abaixo, problem ocorre 96.344 vezes a mais que trouble no corpus, sendo que sua frequência é maior na língua falada (454.50) e sua menor frequência provém da ficção (147.56). Entretanto, é na ficção que a palavra trouble é mais usada (132.36). Gramaticalmente, problem é substantivo e trouble pode funcionar como substantivo e também como verbo: "feelings of homesickness trouble me". 


\section{Revista do SELL}

v. 5 , no. 1

ISSN: 1983-3873

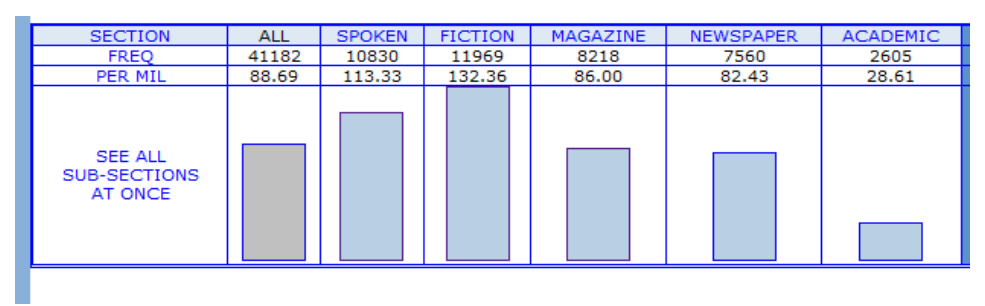

Figura 5: Frequência da palavra trouble no corpus. Fonte: Corpus of Contemporary American English

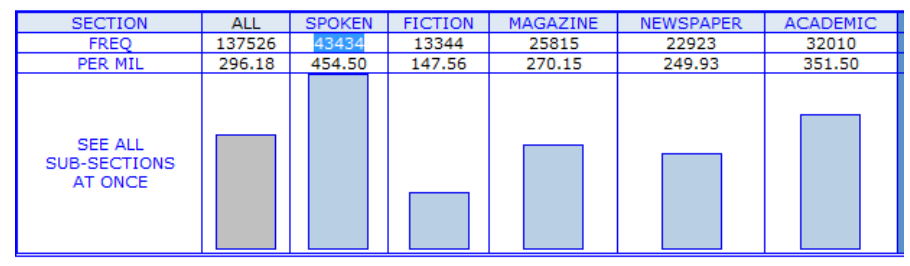

Figura 6: Frequência da palavra problem no corpus. Fonte: Corpus of Contemporary American English

Em relação aos colocados, nos 20 primeiros à esquerda de problem, a ocorrência de adjetivos é dobrada em comparação com trouble. Dois deles foram usados para ambos: big e real. Advérbios como more, much, no e into, somente antecedem trouble nos exemplos. Além de adjetivos da ordem de grandeza ou quantidade (little, big, lot, huge, potencial), os colocados à esquerda demonstram ambiente semântico neutro ou com prosódia semântica negativa comprovada pelas palavras: drug, serious, worse e difficult.

Os verbos antecedentes não se repetem, sendo que para trouble o sentido é de haver, portar um problema (have, get) e para problem os verbos denotam resolução do problema (solve, fix, face, resolve). O fato de os verbos que antecedem trouble apresentarem a partícula -ing não se reproduz com problem. Em relação aos substantivos colocados de trouble, temos somente spot "The debates have been a major trouble spot for him.". Sendo que para problem, um número maior de substantivos pode ser verificado: area, solution, identification, part, etc.

Outro aspecto semelhante, é que with ocorre como colocado à direita para ambos, com informação mútua ${ }^{7}$ e usos parecidos e seguidos de substantivos: "Another problem 


\section{Revista do SELL}

v. 5 , no. 1

ISSN: 1983-3873

with Facebook is that the very qualities that made it so successful as a private firm could sink it as a public one." e "The trouble with tools is that anybody can use them." É importante salientar que, gramaticalmente, ambos podem ocorrer como substantivos contáveis: "Well, to forget all of our troubles, we're going to the Bahamas in 17 days." e "Table 1 shows some of the non-cardiac problems which may be detected."

Para sinônimos de problem, o corpus apresenta: question, problem, difficult, challenge, catch, difficulty, puzzle, problematic, obstacle e tricky, entre outros. Para trouble, temos: work, thought, care, problem, attention, effort, disease, trouble, trial, concern e conflict.

\subsection{Linhas de concordância de trouble}

Por meio das linhas de concordância são observados os ambientes nos quais as palavras surgem. As 50 primeiras linhas de concordância de trouble têm origem nas notícias, Associated Press, New York Times, Atlanta Journal Constitution, Houston Chronicle e Washington Post. Dessas linhas, por 14 vezes preposições antecedem prontamente o substantivo: heap of trouble, about trouble, we're in trouble, got us into trouble. $\mathrm{Na}$ análise do teor dos contextos aparentes, obtemos dados diferentes dos dados gerados pelos colocados. Com o olhar voltado para o contexto no qual as palavras ocorrem, é possível observar um quadro de palavras com prosódia semântica negativa, como attacks, deaths, problems, invasion, extinction, feared, difficulty, obsessive, offender. Os assuntos geram em torno da violência (police, combat), de problemas econômicos (financial, budget, euro, economic) problemas técnicos (engine trouble, installation of engine), com a segurança (Security) e assuntos relacionados à emprego (job), política (Republicans, vote), economia (economic heavyweight) entre outros.

As linhas trazem à sua direita verbos como: began, getting, affording, winning, concluding, holding, remembering, raising, connecting; preposições: in, on, with, from, for, at. Como substantivo, o nódulo aparece no final da frase pelo menos por 9 vezes. Já as preposições que precedem as linhas de concordância são of, about, in, by e into; adjetivos

"Informação mútua é a estatística que indica o grau de força de ligação entre duas palavras. A informação mútua pode ser utilizada para calcular colocações ao indicar a força de relação de co-ocorrência entre um nódulo e um colocado." (MCENERY \& HARDIE , 2012, p. 247) 


\section{Revista do SELL}

v. 5 , no. 1

ISSN: 1983-3873

como financial, obsessive, violent, fanatics e global, verbos como gave,had, bring, caused e feared; substantivos como working-class, incidents, mission e military.

Não há ocorrência significativa de adjetivos como pré-colocados do nódulo e substantivos como: heap, songs, combat e public employers antecedem trouble.

Com base na leitura do contexto expandido da linha 39, abaixo, compreende-se que o sentido de trouble é: "se dar ao trabalho de, não se incomodar com algo":

zape to nide carts stored outdoors, Aldrıch saıd. \# Aldrich encourages resıdents to keep a smaller contaıner nanc It every week. " If it's not full, why go to the trouble of rolling it out? " she said. \# Because the Township can nc ff - like cardboard boxes. You can just break them down and put them in the cart, " she said. \# Single stream ef

Figura XXX: Contexto expandido da palavra trouble:. Fonte: Corpus of Contemporary American English

he says with a tired chuckle. "I'll get into a heap of trouble. " \# Ten years ago, there was a series of dingo attacks on being liquidated "if Silberkleit stays, Goldwater said in court papers. \# The trouble began after the 2007 and 2008 deaths of former Archie Comic leaders Richard Goldwater and as General Motors, Ford Motor Co. and Chrysler Group LLC were heading into financial trouble, they realized that people were shifting away from trucks and sport utility vehicles to the industry. In 1998, J.D. Power and Associates, which surveys owners about trouble with their cars after three years, found an industry average of 278 problems per not wearing a belt. \# " If a kid is prone to getting in trouble and not taking school seriously, then (the fines are) a steep slope including Obama's two picks, Elena Kagan and Sonia Sotomayor, will have no trouble concluding that Congress did not overstep its authority in adopting the insurance requirement that is Santorum retort: "If Mitt Romney's an economic heavyweight, we're in trouble. " \# Aside from a pair of TV interviews, Santorum spent the day lap in Friday's practice. On Saturday, he said the team was having trouble with the installation of an engine.... Ryan Briscoe is the first Australian national were happy with that start, but even a movie aiming for laughs had some trouble making in-roads against " The Avengers. " \# " This is a full-out comedy, She worried that some protesters participated simply " to do stupid things " and cause trouble. \# Some participants called for the dissolution of NATO, the 63-year-old military alliance to three more attempts in the shootout, which begins at 4:30 p.m. \# ENGINE IROUBLE: Though the Lotus engines have been slow all week at Indianapolis, two drivers humanitarian, not combat, mission, and that explains why Washington for years had trouble getting Europeans to provide more forces. \# Also, the U.S. invasion of Iraq . \# The Camp David gathering opened with a Friday evening discussion focused on global trouble spots Iran and Syria. Obama said the session also touched on North Korea's Police after a series of violent incidents in Glasgow. \# Many games bring trouble in Scotland -- the rivalry between Aberdeen and Rangers is particularly sour, and Saturday we hear it' attitude. The police say they know which songs have caused trouble in the past and will be working to focus on those. \# Bobby Simpson of the support and lower-level jobs that free American combat troops to concentrate on the trouble spots. Britain has 9,500 soldiers, the second-largest contingent of troops after the United

Figura 7: Linhas de concordância da palavra trouble no corpus. Fonte: Corpus of Contemporary American English

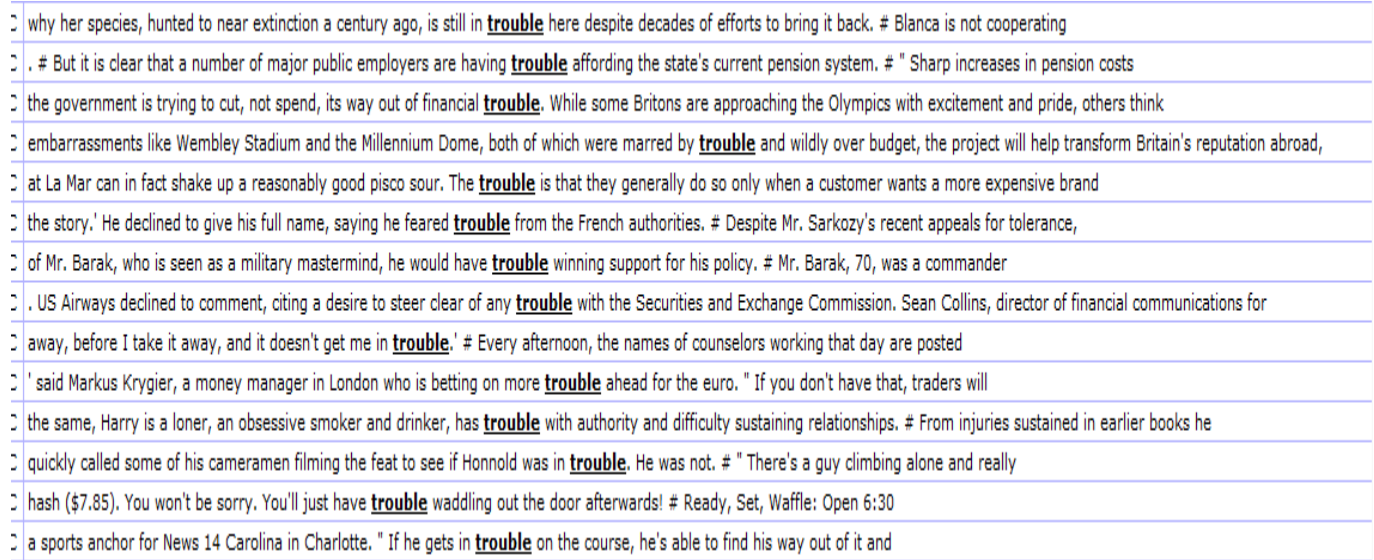

Figura 8: Linhas de concordância da palavra trouble no corpus. Fonte: Corpus of Contemporary American English 


\section{Revista do SELL}

v. 5 , no. 1

ISSN: $1983-3873$

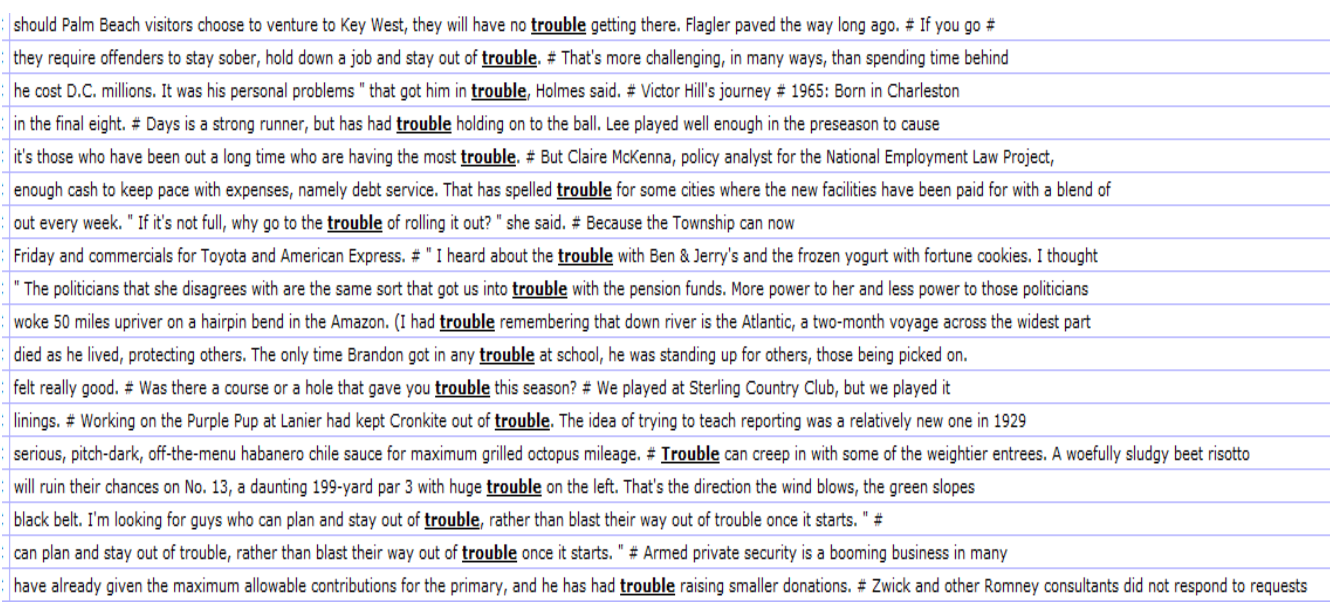

Figura 9: Linhas de concordância da palavra trouble no corpus. Fonte: Corpus of Contemporary American English

\subsection{Linhas de concordância de problem}

As primeiras linhas de ocorrência do substantivo problem são do registro acadêmico, Communications of the ACM, Technology \& Engineering Teacher e Practice Nurse. Os exemplos de uso autêntico da língua mostram que problem é precedido pelo artigo the um número significativo de vezes. Das 50 linhas, 21 delas apresentam o artigo. O uso do artigo definido marca uma condição específica do assunto tratado, ou seja, o uso está atrelado a alguma situação exclusiva "The problem is in determining that BDP".

O contexto em que a palavra surge nas linhas em estudo, de modo geral, diz respeito a problemas funcionais, linguísticos ou relacionados à trabalho: capacity problem, problem solving e network problem. Sinclair (1996), apud O'keeffe et al. (2007) assegura que sentido e forma caminham juntos, uma vez que uma palavra será manifestada em diferentes desenhos estruturais. Como colocados à esquerda do substantivo observa-se o uso de e adjetivos como: old, pervasive, fundamental, poor e main e verbos como: addresses, understand, present, be, suspected, make, solve, captured, surmount, formalize e providing, sendo que o uso de understand foi mais expressivo, que semanticamente aponta para a compreensão, percepção do problema, quando é preciso conhecê-lo.

Antes de problem, temos apenas a ocorrência de uma preposição em: "experimentation in problem solving". Das preposições logo após o substantivo, observase: in, on, to, of, from e by. 


\title{
Revista do SELL \\ v. 5 , no. 1 \\ ISSN: $1983-3873$
}

\begin{abstract}
languages that are directly extensible. Programmers may even be encouraged to think about a problem in terms of a language that would better support the task. This approach is (lambda () desert))))) sign of Racket addresses the problem more directly; it gives programmers tools to explicitly extend the programming language with new Long delays from bufferbloat are frequently misattributed to insufficient bandwidth and this misinterpretation of the problem leads to the wrong solutions being proposed. Congestion is an old prot of the problem leads to the wrong solutions being proposed. Congestion is an old problem on the Internet, appearing in various forms with different symptoms and causing major problems present these problems with their impacts so the community can understand and act upon the problem and, we hope, learn to prevent future problems. This article does not buffering, but it is instead intended to create a wider understanding of the pervasive problem and to give a call to action. internet Buffers and Congestion The latency a continental delay for the U.S. and Europe. Once adequate buffers became routine, another problem could occur: the buffers were now part of the pipe that TCP is so RED manifesto. 2 Note that packet loss for TCP is not in itself a problem but is essential for its functioning in the face of congestion. The excessive and The excessive and consecutive packet losses that come from persistently full buffers do present a problem, which is what the warning drops of AQM prevent (in addition to long a frequent refrain in the Gettys household. When I would attempt to debug the problem, like a willo -the-wisp, it would usually vanish. On several occasions symptoms time on my ISP s support line before they vanished. I attributed the recurring problem to the doubfful quality of the cable to my house or equipment damage from a in immersive teleconferencing, I knew I had to dig into the intermittent poor network problem, if only for myself. An Enlightening Lunch. Suspecting features of my cable An Enlightening Lunch. Suspecting features of my cable provider to be part of the problem, I met with Comcast s Rich Woundy, who provided a number of new Woundy, who provided a number of new issues to consider:.. The big-buffers problem, which David Clark (Internet network architect, currently senior research scientist at MIT that the delays went away when I suspended my large data transfer explained why the problem disappeared when I went looking for it; in order to debug the network, nrder tn dehun the netwnrk $I$ was stnnninn the wnrk that was indurinn the nrnhlem Parket cantures at Hnme and ahrnar I tnnk a ranture nf a larne
\end{abstract}

Figura 11: Linhas de concordância da palavra problem no corpus. Fonte: Corpus of Contemporary American English

order to debug the network, I was stopping the work that was inducing the problem. Packet captures at Home and abroad. I took a capture of a large - Finally, I had collected enough disturbing data to be consistent with the big-buffers problem, and I suspected that the problem was endemic among all technologies and providers. disturbing data to be consistent with the big-buffers problem, and I suspected that the problem was endemic among all technologies and providers. 300400500050010001500 two orders of magnitude so as not to sacrifice bandwidth or latency. Compounding this problem, modern operating systems adjust socketbuffer sizes in response to observed delay; so operating applies at the network edge where a single flow can congest a link. The problem is in determining that BDP. Bandwidth variations of two or more orders of magnitude the operating system, wireless link, and broadband service. Why is overbuffering a problem? Oversized buffers fill and cause delay, destroying many uses of the Internet: , whether remote gaming, hosted desktop systems, or backup. Solving the bufferbloat problem is necessary for seconds their successful deployment. the tip of the iceberg Operating systems and improvement that characterized the early Internet. The first step is to make the problem apparent. Consumer tests are important (for example, Speedtest.net, Sam-Knows, M-Labs congestion windows to grow too slowly, which degrades throughput significantly. To solve this problem, some researchers have proposed offloading more TCP functionality to the hypervisor. Ano current research in $\mathrm{I} / \mathrm{O}$ virtualization is focused on either new interposition functionality that solves some problem or optimizations for reducing the overheads associated with the virtualization. 0 of the biggest challenges of pass-through mode affects devices that use DMA. The fundamental problem is that the driver in the VM will program device DMA using the guest s resides. This is not only incorrect, but also a large safety and security problem since the device could read and write memory potentially belonging to the hypervisor or some to $\$ 3$ billion per year 25 of direct losses to victims in the U.S. The main problem is a lack of data from banks and other institutions that suffer losses; as presentations on the economics of computer security, Cormac Herley of Microsoft Research captured the problem succinctly, asking: What is the first thing you think of when you hear in warnings, support for properly identifying Web sites, and authentication. A general problem with security warnings is that users often close them the instant they appear, a rational behavior, as many warnings are so obtuse people don $\mathrm{t}$ understand what the problem is or what they should do. Other warnings annoyingly contributed articles interrupt what people

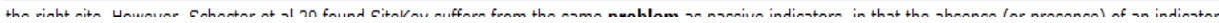

Figura 12: Linhas de concordância da palavra problem no corpus. Fonte: Corpus of Contemporary

\section{American English}

\footnotetext{
$\mathrm{C}$ the right site. However, Schecter et al.29 found SiteKey suffers from the same problem as passive indicators, in that the absence (or presence) of an indicator

C. Since any communication medium can be used for phishing, it is also a problem that can never be solved. The best we can hope for is to blunt

C jakobsson, M. and Myers, s. Phishing and Countermeasures: Understanding the Increasing Problem of Electronic Identity Theft. wiley-Interscience, 2006. 21. keizer, g. California enacts

$\mathrm{C}$ to the question of how to guarantee longterm confidentiality, the most challenging unaddressed open problem from previous works. In this article, we formalize the problem by defining upgrading

C challenging unaddressed open problem from previous works. In this article, we formalize the problem by defining upgrading all PKi instances is systemically and logistically costly since PKi structures

C from updating or renewing cards within a reasonable amount of time. To surmount this problem we must contend with the bottleneck of infrastructure, as well as update existing PKI

$\mathrm{C}$ In the following sections, we propose a cost-efficient concept to mitigate the current PFW problem within PKI and maintain security for longer periods than originally envisioned. asymmetric secrecy P

C will exist from 2019 to 2027. PUB in Step 3 suffers from the same problem. When it occurs, the scheme will not provide long-term confidentiality. Figure 3

C that capacity is exhausted, additional servers can scale the service and solve the capacity problem in a centralized manner. Conclusion Security of cryptographic algorithms is the most important eler

$\mathrm{C}$ items and medicine containers. Great progress has been made in providing solutions to this problem by harnessing $\mathrm{OCR}$, which has become a mature and mainstream technology after decades of

C such as an image of a restaurant sign captured from across the street. The problem of text detec $b$ http: //knfbreader.com c http: **34;23325;TOOLONG **36;23361;TOOLONG figure 2. experiments

$\mathrm{C}$ motion blur), and low contrast due to poor illumination. A closely related problem is finding and recognizing signs 24 characterized by non-standard fonts and layouts that may encode important

C, an alternative approach to package identification is to treat it as an object recognition problem, 38 which has the benefit of not requiring the user to locate the barcode

$\mathrm{C}$ accuracy to distinguish between important mathematical properties at nearby pixels in the prompt. This problem is figure 3. 3D models. the 12 models from our study, each shown

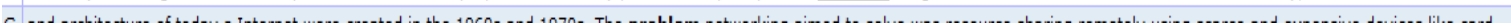

Figura 13: Linhas de concordância da palavra problem no corpus. Fonte: Corpus of Contemporary American English

3.6 Comparação das linhas de concordância de trouble e problem 


\section{Revista do SELL \\ v. 5 , no. 1 \\ ISSN: 1983-3873}

Biber, Conrad \& Reppen (1998) afirmam que a partir das linhas de concordância são revelados os diferentes significados das palavras. Além disso, é possível inferir a partir delas como as palavras podem ser apropriadas para diferentes contextos.

A comparação dos dois nódulos em estudo comprova que o uso pode se caracterizar de maneira diferente. Para exemplificar, as preposições in e into foram usadas com trouble nas linhas de concordância por cerca de 8 vezes, com o sentido de estar em apuros, ou ter sido colocado diante de uma situação difícil, "It was his personal problems that got him in trouble, Holmes said".

Isso não ocorre com problem, que ao contrário de trouble, também não aparece altamente cercada de palavras com conotação negativa (attacks, deaths, problems, invasion, extinction, feared, difficulty, obsessive, combat), o que evidencia que a prosódia semântica também é distinta. O contexto semântico de trouble corrobora o efeito de sentido da palavra e realça esse cenário. A conotação desfavorável é perceptível por meio do conjunto de colocados (PARTINGTON,1998). O'keeffe, Mccarthy e Carter (2007) afirmam que algumas palavras, frases e construções tornam-se associadas a alguns significados devido à coocorrência com palavras de determinada categoria semântica. Sendo assim, é possível avaliar que problem possui prosódia semântica neutra. Notoriamente, várias preposições antecedem trouble, o que não ocorre com problem. $\mathrm{A}$ frequência da expressão understand the problem no corpus é de 91 vezes, ao passo que a expressão understand the trouble ocorre apenas 3,"We've had this experience; we now

\section{understand the problem".}

Diante do exposto, é possível comprovar que em conjunturas formais ou institucionais, há a tendência inclinada para o uso de problem. O nódulo também ocorre na ficção, mas é lá que há menor ocorrência de problem, comparado aos outros registros, ao passo que para trouble, é no contexto da ficção que obteve maior frequência. Por meio das análises verifica-se que quando se trata de alguma situação de adversidade, em que a pessoa está em apuros, sofre alguma ameaça, está em risco ou teve algum contratempo, usa-se trouble. Já o uso de adjetivos posicionados à esquerda é mais significativo com problem, "It was his personal problems "that got him in trouble, Holmes said.".

Mesmo por meio desta breve análise é possível constatar que os padrões de uso de trouble e problem se diferenciam e demonstram que as palavras não podem ser 


\section{Revista do SELL}

v. 5 , no. 1

ISSN: 1983-3873

usadas intercambiavelmente. Essa diferença explica porque as palavras foram usadas em números diferentes nos diferentes registros. Problem, palavra de ambiente léxico neutro, é usada com maior frequência em textos acadêmicos e para assuntos gerais. Por outro lado, trouble, que aparece precedido de adjetivos por mais vezes e ocorre cercado de palavras com prosódia negativa, é a preferida pela ficção.

\section{Resultados}

A Linguística de Corpus conta com importantes instrumentos para que o estudo da língua com base em dados seja feita. As implicações da presente pesquisa corroboram o pressuposto de Sinclair (1991), pois cada uma das palavras em análise (problem e trouble) pode ocorrer com sentidos diferentes associadas a um conjunto distinto de padrões.

"Quando se diz que a variação não é aleatória, na verdade, está se afirmando que a linguagem é padronizada ('patterned'). A padronização se evidencia pela recorrência, isto é, uma colocação, coligação ou estrutura, que se repete significativamente, mostra sinais de ser na verdade um padrão lexical ou léxico-gramatical." (SARDINHA, 2000, p. 29)

Verificamos em trouble a demonstração de uma associação atrelada a palavras de cunho negativo e mais diverso e representa a opção mais informal, referenciada pelos dados do corpus posto que, "muitos usos de palavras e frases mostram a tendência de ocorrerem em um certo ambiente semântico"8 (SINCLAIR, 1991, p.112).

Os usuários da língua, sejam nativos ou estrangeiros, têm a seu favor a demonstração dos usos pré-estabelecidos como direcionamento, em razão de que padrões são replicados no uso autêntico da língua. Os resultados da presente pesquisa baseada em corpus atestam que as diferenças de uso não são aleatórias.

\section{Considerações finais}

Por meio desse estudo averiguamos o uso das palavras trouble e problem, comparamos seus colocados e algumas linhas de concordância, as quais nos permitem

8 (SINCLAIR, "many uses of words and phrases show a tendency to occur in a certain semantic environment" 1991 , p.112). 


\section{Revista do SELL}

v. 5 , no. 1

ISSN: 1983-3873

observar em quais situações as palavras ocorrem. O material oferecido pelo corpus oferece uma extensa lista de ocorrências e contextos (BIBER; CONRAD \& REPPEN, 1998). Mesmo nos detendo às 50 primeiras linhas para cada palavra, avaliamos que o recorte foi profícuo para atender aos objetivos selecionados, pois semelhanças e diferenças entre os nódulos foram descritas e ainda, foi possível chegar ao entendimento de que, em resposta à nossa pergunta de pesquisa, os termos não podem ser usados como sinônimos em múltiplos contextos.

Entendemos que os estudos baseados em corpora fomentam discussões autênticas e têm o potencial de promover pesquisas inovadoras, uma vez que a observação dos dados obtidos pelo uso autêntico da língua permite a análise de construções léxico-gramaticais não pensadas anteriormente e, na maioria das vezes, não abordadas nos livros didáticos.

Ao ensinar o vocabulário, o professor de língua estrangeira tem em mãos uma ferramenta que pode auxiliá-lo no cotidiano de diferentes maneiras, uma delas foi abordada no presente estudo, que, como vimos, aponta, dentre outros aspectos, que palavras ditas sinônimas possuem diferenças relevantes no uso, e que uma mesma palavra possui vários significados e não possuem sentido sozinhas, pois se relacionam para gerar significado. De acordo com McEnery e Hardie (2012), a Linguística de Corpus é capaz de reorientar a abordagem do estudo de uma língua.

Gostaria de encerrar esta discussão com Lewis (1997), que fez uma interessante analogia para tratar dos colocados ao dizer que pessoas encontram-se com outras que nunca viram anteriormente e, algumas vezes, tornam-se bons amigos, mas outras, nunca mais se veem novamente. Procuramos estar com algumas pessoas mais que com outras. O mesmo ocorre com as palavras, as quais são geralmente encontradas num mesmo ambiente léxico ou gramatical e coocorrem com regularidade mais ou menos frequente. Os colocados fornecem aos aprendizes um princípio de organização poderosa da língua.

\section{Sugestão para Pesquisas Futuras}

Foram consideradas aqui as palavras trouble e problem, todavia, uma nova investigação pode ser feita considerando as palavras trouble, problem e matter, considerando que a palavra matter também pode ter uma conotação próxima das outras 


\section{Revista do SELL}

v. 5 , no. 1

ISSN: 1983-3873

em alguns contextos, mas diferenças no uso. Além disso, uma pesquisa com base nos cognatos problem e problema pode constatar a correspondência ou dessemelhança na utilização dos termos.

Um estudo propício para análise pode ser feito a respeito do uso de trouble e problem em corpora de aprendizes, com o intuito de investigar se há uma frequência maior no uso do cognato, a tendência de uso dos estudantes e o porquê das escolhas.

\section{Referências}

ALMEIDA, V. C. Investigando Colocações em um corpus de Aprendiz. Belo Horizonte: 2014. 165 f. Tese (Doutorado em Linguística Aplicada) - Programa de Pós-Graduação em Estudos Linguísticos, Faculdade de Letras, Universidade Federal de Minas Gerais, Belo Horizonte, 2014.

BARLOW, M. Software for corpus access and analysis. In: SINCLAIR, J. (org) How to use corpora in the language teaching. Amsterdam: John Benjamins. 2004.

BENNETT, G. Using corpora in the language learning classroom: corpus linguistics for teachers. Michigan: University of Michigan Press, 2010.

BIBER, D.; CONRAD, S.; REPPEN, R. Corpus Linguistics: Investigating language structure and use. Cambridge: Cambridge University Press, 1998.

DAVIES, M. Corpus of Contemporary American English (COCA). 2008. Disponível em: <http://corpus.byu.edu/coca/>. Acesso em: 10 nov. 2014.

DUTRA, D. P. Conscientização Linguística com base em corpora online. Revista Intercâmbio. São Paulo: LAEL/PUC-SP, v. XX, p. 79-98, 2009.

HUNSTON, S. Corpora in applied linguistics. Cambridge: Cambridge University Press. 2002.

LEWIS, M. Implementing the lexical approach: Putting Theory into Practice. Hove, England: Language Teaching Publications, 1997.

MCENERY, T.; HARDIE, A. Corpus Linguistics: Method, Theory and Practice. Cambridge: Cambridge University Press, 2012.

PARTINGTON, A. Patterns and Meanings: using corpora for English language research and teaching. Amsterdã/Filadélfia, John Benjamins, 1998.1-14,29-47, 48-78.

O'KEEFFE, A.; MCCARTHY, M.; CARTER, R. From corpus to classroom: language use and language teaching. Cambridge; New York: Cambridge University Press, 2007.

REPPEN. R. Using Corpora material in the classroom and creating corpora for class use. In: Using Corpora in the language classroom. New York: Cambridge University Press, 2010. 


\section{Revista do SELL}

v. 5 , no. 1

ISSN: $1983-3873$

RÖMER, Ute. Corpus research and practice: What help do teachers need and what can we offer? In: Aijmer, Karin (ed.). Corpora and language teaching, Aijmer, Karin (ed.), 8398, 2009.

SARDINHA. T.B. Linguística de Corpus: Histórico e Problemática. Delta Vol 16, № 2, 2000. 323-367.

SARDINHA. T.B. Linguística de Corpus. Barueri, SP: Editora Manole. 2004.

SARDINHA, T.B. Metáforas e Linguística de Corpus: metodologia de análise aplicada a um gênero de negócios. DELTA vol.27 no.1 São Paulo, 2011.

SINCLAIR, J. Corpus, Concordance, Collocation. Oxford: Oxford University Press, 1991.

TSUI. A. B. M. What teachers have always wanted to know- and how corpora can help. In: SINCLAIR, J. (org) How to use corpora in the language teaching. Amsterdam: John 